\title{
FBXL5 Inhibits Metastasis of Gastric Cancer Through Suppressing Snail1
}

\author{
Weidong Wu ${ }^{a}$ Honghua Ding ${ }^{b}$ Jun $\mathrm{Cao}^{a}$ Weihao Zhang \\ aDepartment of Gastrointestinal Surgery, Shanghai JiaoTong University affiliated First People's Hospital \\ (North), bepartment of Oncology, Shanghai JiaoTong University affiliated First People's Hospital \\ (North), 'Department of Gastrointestinal Surgery, Shanghai JiaoTong University affiliated First People's \\ Hospital (South), Shanghai, China
}

\section{Key Words}

FBXL5 • Gastric cancer • Snail1 • Cancer invasion • Metastasis

\begin{abstract}
:
Background/Aims: The Snail family of transcription factors controls epithelial to mesenchymal transition (EMT), a process associated with tumorigenesis originated from epithelial cells. Snail1 is a member from Snail family and upregulation of Snail 1 has been detected in gastric cancer (GC), suggesting a potential role of Snail1 in GC metastasis. We have recently reported that FBXL5 regulates cortactin by inducing its ubiquitylation and subsequent proteasomal degradation, resulting in inhibition of metastasis of GC. However, a role of FBXL4 in regulation of other EMT-associated proteins is not unknown.Methods: The levels of FBXL5 and Snail1 as well as their relationship were determined in GC specimen. Co-immunoprecipitation (IP) was performed to detect the interaction between Snail1 and FBXL5 in GC cells. The effects on Snail1 by FBXL5 were examined by overexpression of depletion of FBXL5 in GC cells. The invasiveness of the FBXL5-modified GC cells was examined in both scratch wound healing assay and transwell matrix penetration assay. Results: FBXL5 also physiologically interacted with Snail1. FBXL5 inhibited Snail1 to suppress GC cell invasiveness. Conclusion: FBXL5 negatively regulates several EMT-enhancing factors. FBXL5 is an attractive novel target for inhibiting invasion and metastasis of GC cells.
\end{abstract}

Copyright $(2015$ S. Karger AG, Basel

\section{Introduction}

Gastric cancer (GC) is the fourth most common malignant human cancer and the second leading cause of death worldwide, with an incidence of nearly one million per year [1-4]. W. Wu and H Ding contributed equally to this work.

Weihao Zhang

KARGER 125
Department of Gastrointestinal Surgery, Shanghai JiaoTong University Affiliated First People's Hospital (South), 650 Xinsongjiang Rd, Shanghai 201620 (China)

Tel. +862137798571, E-Mail weihao_zhang14@163.com 
The majorities of the subjects diagnosed with GC already have developed metastasis or are developing metastasis [1-4], which highlights the importance of the elucidation of the molecular control of migration and invasiveness of GC cells.

The epithelial-mesenchymal transition (EMT) is a process by which epithelial cells acquire a migratory, mesenchymal phenotype, as a result of its repression of E-cadherin [57]. EMT is essential for numerous physiological and pathological processes in development, in wound healing, in tissue fibrosis and remodeling, and in initiation of tumor metastasis [5-7]. Several signaling pathways (TGF-beta, FGF, EGF, HGF, Wnt/beta-catenin and Notch) induce EMT [8-19]. Loss of E-cadherin is considered to be a fundamental event in EMT [57]. Transcription factors that repress E-cadherin are considered as EMT-promoting factors, including Snail1, Slug1, ZEB1, ZEB2, E47 and Kruppel-like factor 8 (KLF8) that directly bind to E-cadherin promoter and repress its transcription, and including Twist, TCF4, SIX1 and FOXC2 that repress E-cadherin indirectly [5-7]. Snail1 is a member of the Snail superfamily of zinc-finger transcription factors [20,21]. Snail 1 is composed of 264 amino acids and usually acts as a transcriptional repressor. Phosphorylation and nuclear localization of Snail1, governed by PI3K and Wnt signaling pathways crosstalk, are critical in Snail1's regulation $[20,21]$. Snail1 has a pivotal role in the regulation of EMT, involving the loss of E-cadherin and claudins with concomitant upregulation of vimentin and fibronectin, among other biomarkers [20, 21]. Recently, it is found that Snail1 degradation by FBX011 is dependent on Ser-11 phosphorylation of Snail by protein kinase D1 (PKD1), and FBX011 blocks Snailinduced EMT, tumor initiation, and metastasis in multiple breast cancer models [22].

FBXL5 is an F-box protein, a member of the F-box protein family characterized by the F-box motif comprised of about 40 amino acids [23-30]. The F-box proteins are major components of ubiquitin protein ligase complex SKP1-cullin-F-box (SCFs), which plays a critical role in phosphorylation-dependent protein ubiquitination [23-30]. Particularly, FBXL5 is an iron sensor, which promotes IRP2 ubiquitination and then its degradation [23-30]. Recently, we reported that FBXL5 mediates the ubiquitination and degradation of cortactin, which is necessary for the invasion and metastasis of GC cells [31]. However, whether FBXL5 may regulate other EMT-related proteins is so far unknown. Here we showed that FBXL5 also physiologically interacted with and decreased the levels of Snail1 to inhibit gastric cancer cell invasiveness.

\section{Materials and Methods}

Patient tissue specimens

Paired GC specimen ( 9 without metastasis and 11 with metastasis) and normal gastric tissue (NGT) from 20 GC patients were collected for this study. All specimens had been histologically and clinically diagnosed at the Department of Gastrointestinal Surgery, Shanghai JiaoTong University affiliated First People's Hospital from 2007 to 2013. For the use of these clinical materials for research purposes, prior patient's consents and approval from the Institutional Research Ethics Committee were obtained.

Cell lines

Human GC cell lines SNU-5 and AGS were both purchased from American Type Culture Collection (ATCC, Rockville, MD, USA). Both cell lines were maintained in Dulbecco's modified Eagle's medium (DMEM, Invitrogen, Carlsbad, CA, USA) supplemented with 15\% fetal bovine serum (FBS; Sigma-Aldrich, St Louis, MO, USA). All cell lines were incubated in a humidified chamber with $5 \% \mathrm{CO} 2$ at $37{ }^{\circ} \mathrm{C}$. For inhibition of ubiquitination by FBXL5, 50 mol// MG132 (Sigma-Aldrich) was applied.

\section{Cell transfection}

The construct and antisense for FBXL5 were purchased from GeneCopoeia (Rockville, MD, USA), and Dharmacon (Lafayette, CO, USA), respectively, as has been described before [31]. For cell transfection experiments, GC cells were grown to 70 to $80 \%$ confluence in 6-well plates. Transfection was performed using the Lipofectamine 2000 according to the manufacturer's instructions (Invitrogen).

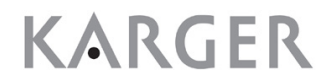




\section{Scratch wound healing assay}

Scratch wound healing assay was performed as has been described previously [32]. Cells were seeded in 24-well plates at a density of $10^{4}$ cells/well in complete DMEM and cultured to confluence. The cell monolayer was serum starved overnight in DMEM prior to initiating of the experiment. Confluent cell monolayer were then scraped with a yellow pipette tip to generate scratch wounds and washed twice with media to remove cell debris. Cells were incubated at $37^{\circ} \mathrm{C}$ for 24 hours. Time lapse images were then captured after 12 hours. Images were captured from five randomly selected fields in each sample, and the wound areas are calculated by NIH ImageJ software.

\section{Transwell migration assay}

Cell migration analysis was performed using a Fluorometric Cell Migration Assay kit with polycarbonate membrane inserts (5- $\mu \mathrm{m}$ pore size; Cell Biolabs, San Diego, CA, USA), according to the manufacturer's protocol. Digital images were obtained from the membranes. The migration cells were stained by CyQuant GR dye (Invitrogen), and then quantified in five randomly selected fields in each membrane, and the average value was defined as an invasion index on three independent membranes.

\section{Cell proliferation assay}

For assay of cell proliferation, pretreated cells were seeded into 96 well-plate at 5000 cells per well and subjected to a Cell Proliferation Kit (MTT, Roche, Nutley, NJ, USA), according to the instruction from the manufacturer.

RNA extraction, reverse transcription and quantitative RT-PCR

Total RNA was extracted from the cultured cells using RNeasy kit (Invitrogen), according to the manufacturer's instruction. For mRNA analysis, complementary DNA (cDNA) was randomly primed from $2.0 \mu \mathrm{g}$ of total RNA using the Omniscript reverse transcription kit (Qiagen, Hilden, Germany). Quantitative Real-time PCR (RT-qPCR) was subsequently performed in duplicate with a 1:4 dilution of cDNA using the Quantitect SyBr green PCR system (Qiagen) on a Rotorgene 6000 series PCR machine. Data were collected and analyzed using the Rotorgene software accompanying the PCR machine. Relative expression levels were determined using the comparative quantification feature of the Rotorgene software. Levels of gene transcripts were normalized to $\alpha$-tubulin, and then compared to controls.

\section{Co-Immunoprecipitation}

Cells were treated with $1 \mathrm{ml}$ of extraction buffer (10 mmol/l HEPEs, pH7.5, $100 \mathrm{mmol} / \mathrm{l} \mathrm{NaCl}, 1$ mmol/l EDTA, 10\% Glycerol, 0.5\% Triton X-100 and $5 \mu \mathrm{mol} / \mathrm{l}$ MG132). The Co-immunoprecipitation (Co-IP) procedure was described previously [31]. Both primary antibodies anti-Snail1 and anti-FBXL5 are generated from rabbits (Cell Signaling, San Jose, CA, USA). Co-IP procedures were performed at $4^{\circ} \mathrm{C}$ unless otherwise indicated, using a Pierce spin column which can be capped and plugged with a bottom plug for incubation or unplugged to remove the supernatant by centrifugation at $1000 \mathrm{~g}$ for 1 minute. The binding of the first antibody to protein $\mathrm{A} / \mathrm{G}$ agarose was performed with the protocol described in Pierce crosslink immunoprecipitation kits (Thermo Scientific, Rockford, IL, USA) with slight modification. For co-IP experiment without using DSS crosslinking, the protein A/G agarose was incubated either 10 $\mu \mathrm{l}$ anti-Snail 1 or $10 \mu \mathrm{l}$ anti-FBXL5 antibody at $25^{\circ} \mathrm{C}$ for $1 \mathrm{~h}$ on a mixer, followed by incubation with $600 \mu \mathrm{l}$ pre-cleared lysate overnight. The immunoprecipitated products were washed with the washing buffer five times and eluted with $2 \mathrm{X}$ Laemmli buffer at $100^{\circ} \mathrm{C}$ for $10 \mathrm{~min}$. The cap of the spin column was loose to avoid overpressure and leakage from the bottom when boiling. The eluting complex was subjected to SDS-PAGE separation for Western blot.

\section{Western blot/immunoblot}

Protein was extracted from the cultured cells by RIPA buffer (Sigma-Aldrich) for Western Blot or got from IP. The supernatants were collected after centrifugation at $12000 \times \mathrm{g}$ at $4^{\circ} \mathrm{C}$ for $20 \mathrm{~min}$. Protein concentration was determined using BCA protein assay, and whole lysates were mixed with $4 \times$ SDS loading buffer (125 $\mathrm{mmol} / \mathrm{l}$ Tris- $\mathrm{HCl}, 4 \% \mathrm{SDS}, 20 \%$ glycerol, $100 \mathrm{mmol} / \mathrm{l}$ DTT, and $0.2 \%$ bromophenol blue) at a ratio of 1:3. Samples were heated at $100{ }^{\circ} \mathrm{C}$ for $5 \mathrm{~min}$ and were separated on SDS-polyacrylamide gels. The separated proteins were then transferred to a PVDF membrane. The membrane blots were first probed with a primary 
antibody. After incubation with horseradish peroxidase-conjugated second antibody, autoradiograms were prepared using the enhanced chemiluminescent system to visualize the protein antigen. The signals were recorded using X-ray film. Primary antibodies for Western Blot are anti-FBXL5 (pTBR1), anti-Snail1 and anti- $\alpha$-tubulin (all purchased from Cell Signaling, San Jose, CA, USA). Secondary antibody is HRP-conjugated anti-rabbit (Jackson Labs, Bar Harbor, ME, USA). Images shown in the figure were representative from 5 repeats. Densitometry of Western blots was quantified with NIH ImageJ software.

\section{Statistical analysis}

Each experiment condition contains 5 repeats. All values are depicted as mean \pm standard deviation and are considered significant if $\mathrm{p}<0.05$. Statistic method: one-way ANONA with a Bonferoni correction. Bivariate correlations were calculated by Spearman's rank correlation coefficients.

\section{Results}

Inverse correlation of FBXL5 and Snail1 in GC specimen

A recent study suggests that FBXL5 may induce nuclear ubiquitination of Snail1 to inhibit EMT [30], and we have preciously shown that FBXL5 mediates the ubiquitination and degradation of cortactin, which is necessary for the invasion and metastasis of GC cells [31]. In order to find out whether FBXL5 may also regulate Snail1 in GC, we examined the FBXL5

Fig. 1. Inverse correlation of FBXL5 and Snail1 in GC specimen. The FBXL5 and Snail1 levels in the GC specimen were examined by Western blot, compared with the paired normal gastric tissue (NGT) from the same patient. (A) We found that GC had significantly lower levels of FBXL5, which was further lower in GC with metastasis (ms). (B) GC had
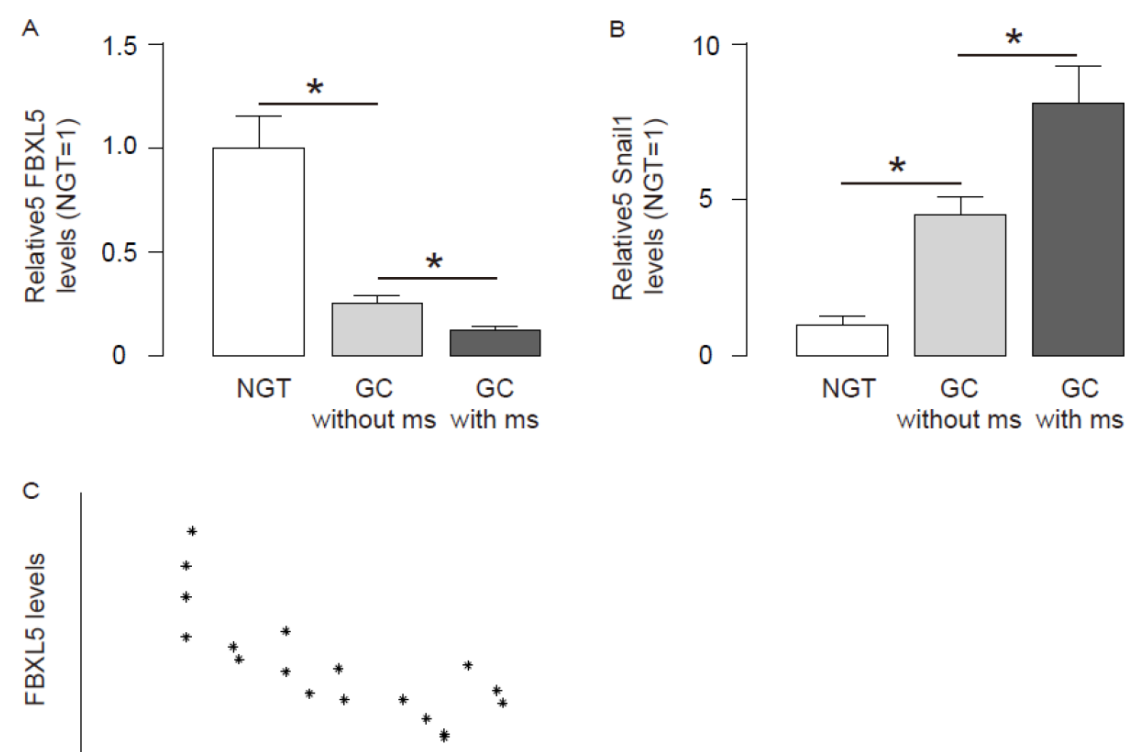

$R=-0.78 p<0.0001$

significantly higher levels of Snail1, which was further higher in GC with metastasis. (C) Strong inverse correlation was detected between FBXL5 and Snail1 $(\mathrm{R}=-0.78 ; \mathrm{p}<0.0001) .{ }^{*} \mathrm{p}<0.05$. $\mathrm{n}=20$. Statistics: one-way ANOVA with a Bonferoni correction. Bivariate correlations were calculated by Spearman's rank correlation coefficients.

Fig. 2. FBXL5 and Snail1 physically associated with each other in GC cells. Co-Immunoprecipitation (CoIP) was performed to detect the interaction between endogenous proteins independently in two GC lines, SNU-4 and AGS. Our results showed that endogenous Snail1 co-precipitated with FBXL5 and vice versa in both lines. IB: immunoblot. IP: immunoprecipitation.

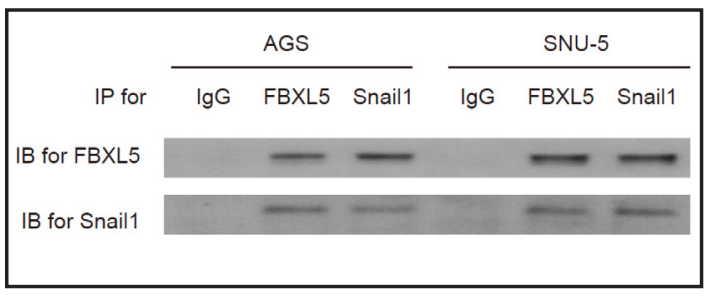




\section{Cellular Physiology Cell Physiol Biochem 2015;35:1764-1772

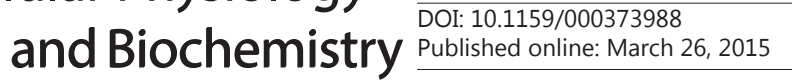

Fig. 3. FBXL5 inhibited Snail1 in GC cells. (A) GC cells were transfected with either a FBXL5-overexpressing plasmid, or a plasmid carrying short hairpin small interfering RNA (shRNA) for FBXL5 (shFBXL5), to increase or decrease FBXL5 levels in GC cells, respectively. GC cells were also transfected with a plasmid carrying a scrambled sequence (scr) as controls. All plasmids contained a GFP reporter to allow visualization of transfected cells. (B-C) The changes in FBXL5 in AGS cells were confirmed by RT-qPCR (B), and by Western blot (C). (D-E) The changes in FBXL5 in AGS cells were confirmed by RT-qPCR (D), and by Western blot $(E) .{ }^{*} p<0.05$. NS: non-significant. $n=5$. Statistics: one-way ANOVA with a Bonferoni correction.

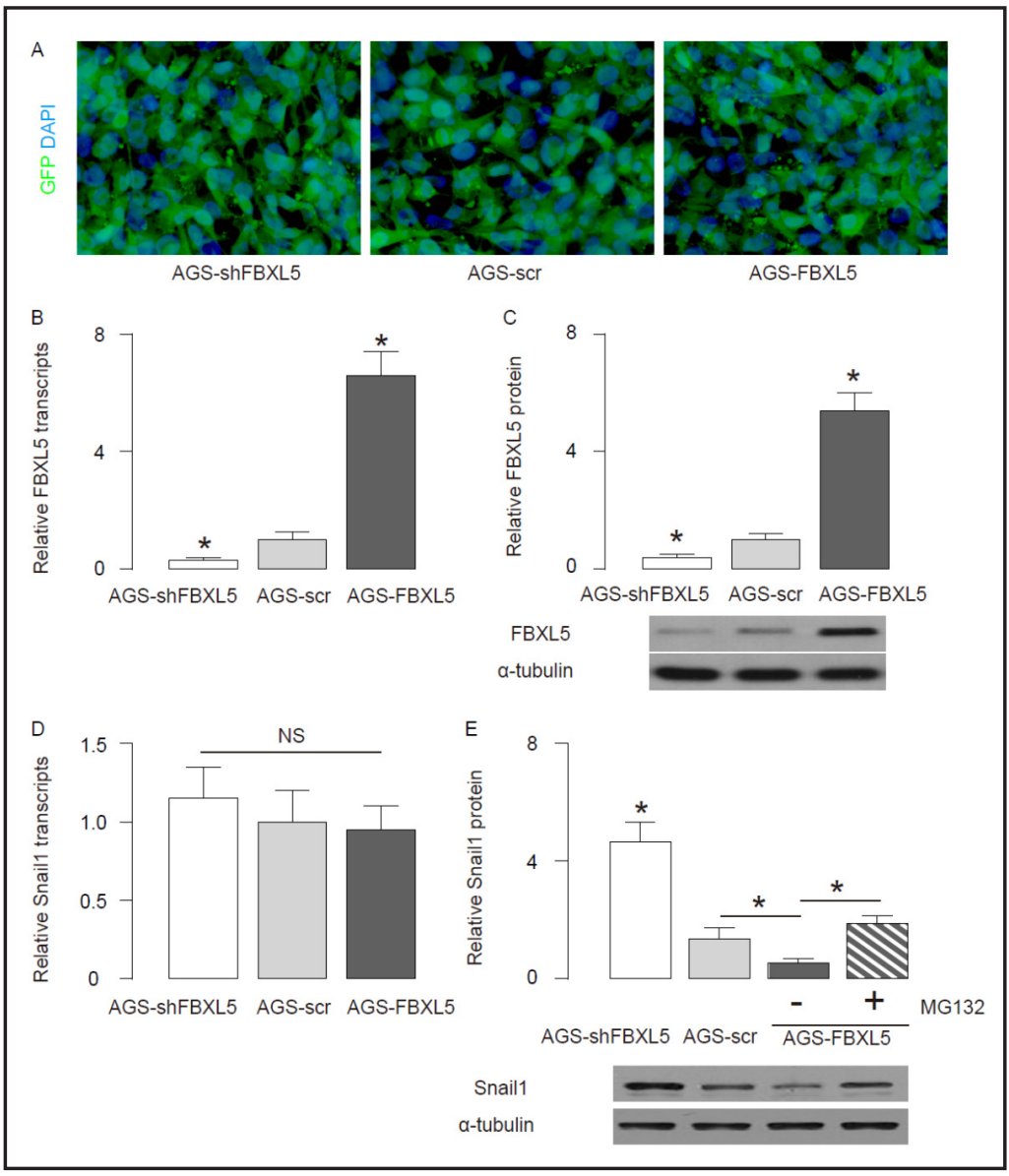

and Snail1 levels in the GC specimen by Western blot, compared with the paired normal gastric tissue (NGT) from the same patient. We found that GC had significantly lower levels of FBXL5 (Fig. 1A, p<0.05), which was further lower in GC with metastasis (ms) (Fig. 1A, $p<0.05$ ). Moreover, GC had significantly higher levels of Snail1 (Fig. 1B, $p<0.05$ ), which was further higher in GC with metastasis (Fig. 1B, p<0.05). Further, Strong inverse correlation was detected between FBXL5 and Snail1 (Fig. 1C, R=-0.78; $p<0.0001$ ), suggesting a possibility of presence of a FBXL5/Snail1 regulatory loop.

FBXL5 and Snail1 physically associated with each other in GC cells

To confirm the specific binding between FBXL5 and Snail1, we performed immunoprecipitation (IP) to detect the interaction between endogenous proteins independently in two GC lines, SNU-4 and AGS. Our results showed that endogenous Snail1 co-precipitated with FBXL5 and vice versa in both lines (Fig. 2). These data suggest that FBXL5 and Snail1 physically associate with each other in GC cells.

\section{FBXL5 inhibited Snail1 in GC cells}

To examine whether FBXL5 may regulate the levels of Snail1 in GC cells, we then transfected the GC cells with either a FBXL5-overexpressing plasmid, or a plasmid carrying short hairpin small interfering RNA (shRNA) for FBXL5 (shFBXL5), to increase or decrease FBXL5 levels in GC cells, respectively. GC cells were also transfected with a plasmid carrying a scrambled sequence (scr) as controls. All the plasmid constructs contained a GFP reporter to allow examination of the transfection efficiency, which is nearly $100 \%$ by all three (Fig. $3 \mathrm{~A}$ ). We analyzed both AGS and SNU-5 cells and got essentially same results within the scope of the current study. Thus, only data from AGS were shown. The changes in FBXL5 in GC cells were confirmed by RT-qPCR (Fig. 3B), and by Western blot (Fig. 3C). We found that neither 


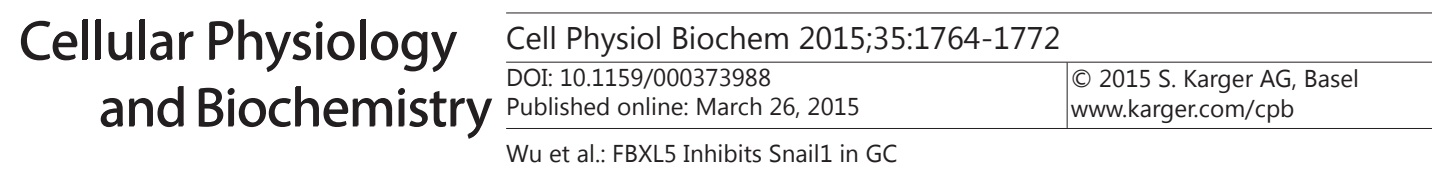

Fig. 4. FBXL5-mediated Snail1 suppression reduced migration and invasion of GC cells in a scratch wound healing assay. The invasiveness of the FBXL5-modified GC cells were examined in a scratch wound healing assay, shown by representative images (A), and by quantification (B). We found that FBXL5-mediated Snail1 suppression reduced migration and invasion of GC cells. ${ }^{*} \mathrm{p}<0.05$. $\mathrm{n}=5$. Statistics: one-way ANOVA with a Bonferoni correction.

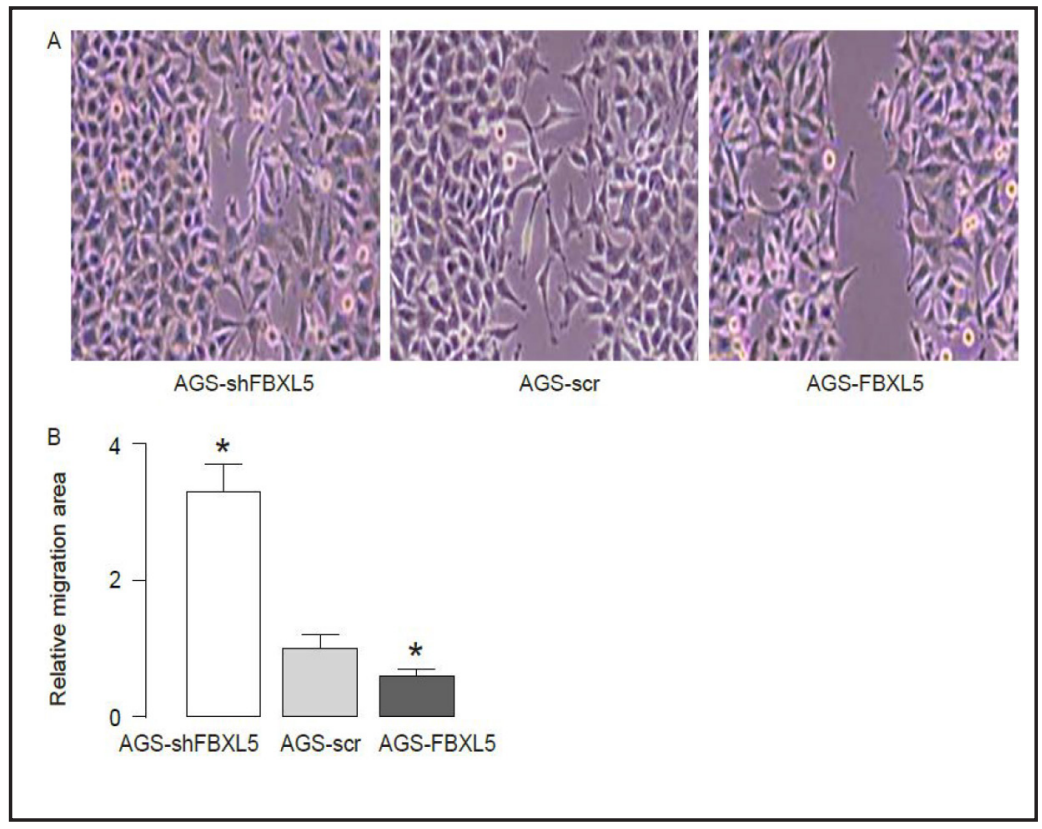

Fig. 5. FBXL5-mediated Snail1 suppression reduced migration and invasion of GC cells in a transwell matrix penetration assay. The invasiveness of the FBXL5-modified GC cells were examined in a transwell matrix penetration assay, shown by representative images (A), and by quantification (B). We found that FBXL5-mediated Snail1 suppression reduced migration and invasion of GC cells. *p<0.05. $n=5$. Statistics: one-way ANOVA with a Bonferoni correction.

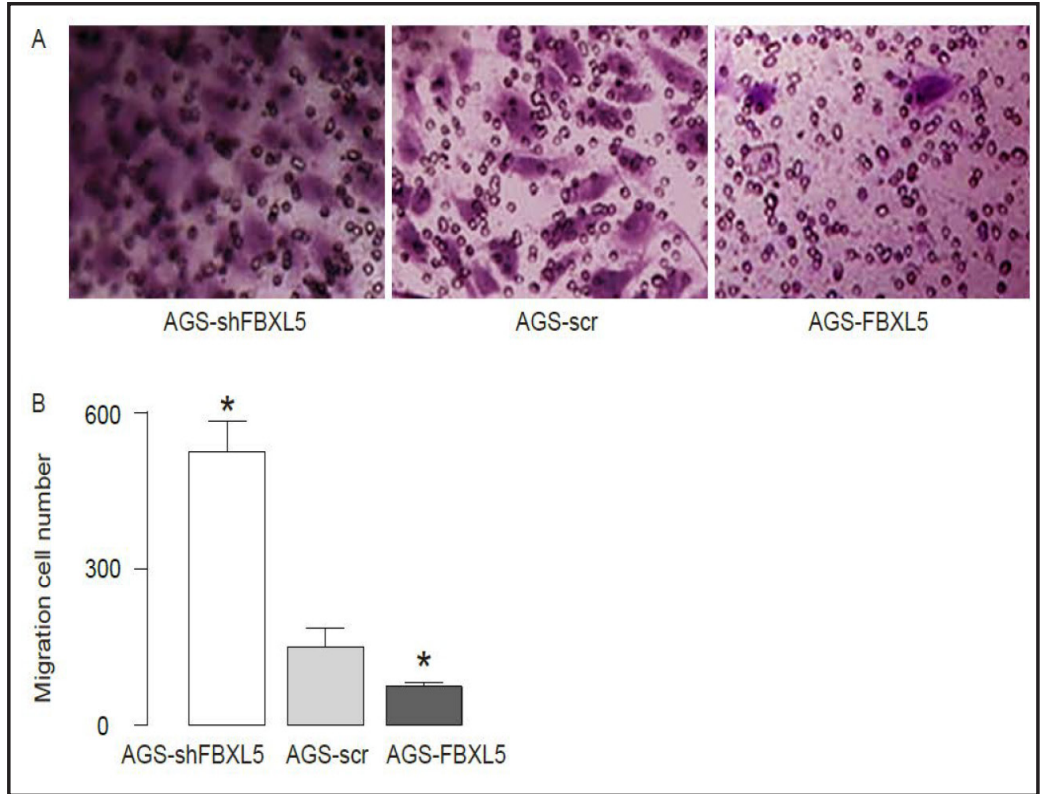

FBXL5 overexpression nor depletion significantly affected the transcript levels of Snail1 (Fig. 3D). However, FBXL5 overexpression significantly decreased the protein of Snail1 in GC cells, while depletion of FBXL5 significantly increased the protein of Snail1 in GC cells (Fig. 3E). Further, a potential ubiquitination inhibitor MG132 significantly rescued FBXL5-dependent decreases of Snail1 protein levels (Fig. 3E). These data suggest that FBXL5 inhibited Snail1 in GC cells at protein level without affecting Snail1 transcription, possibly through enhancing Snail1 ubiquitination-related degradation.

FBXL5-mediated Snail1 suppression reduced migration and invasion of GC cells

Next, we examined the invasiveness of the FBXL5-modified GC cells. We found that FBXL5-mediated Snail1 suppression reduced migration and invasion of GC cells in both a scratch wound healing assay (Fig. 4A-B) and a transwell matrix penetration assay (Fig. 
Fig. 6. MTT assay to examine cell growth. NS: non-significant. $n=5$. Statistics: one-way ANOVA with a Bonferoni correction.

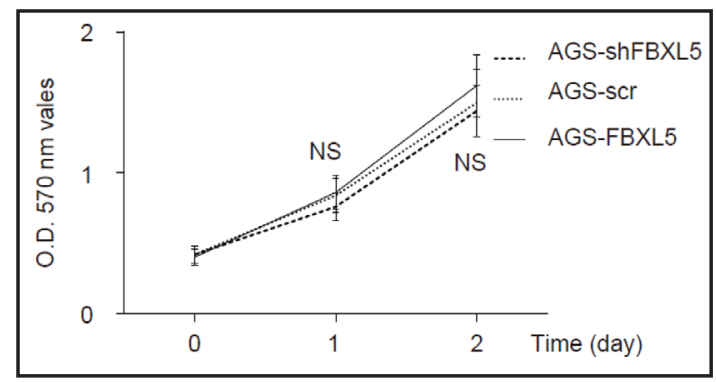

5A-B). Thus, our study suggest that FBXL5 may reduce the invasiveness of GC cells through direct inhibition of Snail1 at protein level. We also examined the cell growth in the FBXL5modified GC cells and found that FBXL5 did not significantly alter cell growth (Fig. 6).

\section{Discussion}

The high malignancy and lethality of GC largely result from its high invasiveness and local and distal metastasis, in which EMT plays a substantial role [1-4]. Among all EMTassociated proteins, Snail1 and cortactin are critical factors. Cortactin is important in promoting lamellipodia formation, invadopodia formation, cell migration, and endocytosis, while Snail1 is a potential EMT activator, and directly inhibited E-cadherin. Although both Snail1 and cortactin promote EMT, there is a recent report showing a negative regulatory effect of Snail1 on cortactin [33]. These data suggest presence of a delicate coordination of these factors in the process of EMT.

FBXL5 is an E3 ubiquitin ligase that target many proteins to regulate their phosphorylation-dependent protein ubiquitination and subsequent degradtion [23-30]. Recently, we reported that FBXL5 mediates the ubiquitination and degradation of cortactin, which is necessary for the invasion and metastasis of GC cells [31]. However, whether FBXL5 may regulate other EMT-related proteins is so far unknown. A recent report showed that FBXL5 has a potential to be a novel Snail1 ubiquitin ligase [30]. FBXL5 interacts with Snail1 in the nucleus to promote its polyubiquitination, decrease its protein stability by impairing DNA binding [30]. FBXL5 is highly sensitive to stress and could be suppressed by iron depletion and $\gamma$-irradiation [30]. These results characterize FBXL5 as a novel nuclear ubiquitin ligase controlling Snail1 protein stability and provide the molecular mechanisms underlying the radiotherapy-enhanced EMT by Snail1. Thus, we were prompted to examine this model in GC.

Our data compellingly show that FBXL5 significantly decreased in GC, and especially in GC with metastasis, and inversely correlated with the Snail1 levels in the patients' specimen. These data provide a clinical relevance of the current study. Moreover, we further showed that FBXL5 physiologically interacted with and decreased the levels of Snail1 to suppress gastric cancer cell invasiveness.

Together with previous reports by us and by others, a comprehensive regulatory network on EMT, highlighting a pivotal role of FBXL5, has been proposed. FBXL5 regulates cortactin through induction of its ubiquitylation, and FBXL5 similarly regulates Snail1. Among all EMTassociated proteins, Snail1 and cortactin are both critical factors. Cortactin is important in promoting lamellipodia formation, invadopodia formation, cell migration, and endocytosis, while Snail1 is a potential EMT activator, and directly inhibited E-cadherin. Although both Snail1 and cortactin promote EMT, there is a recent report showing a negative regulatory effect of Snail1 on cortactin [33]. These data suggest presence of a delicate coordination of these factors in the process of EMT.

Future approaches should include additional gain-of-function and loss-of-function experiments $s$ to confirm the detail of the regulatory machinery determined here in GC to figure out the exact amino acids on Snail1 protein to be ubiquitinated. Thus, our study sheds light on FBXL5 as a promising therapeutic target for GC. 


\section{Cellular Physiology Cell Physiol Biochem 2015;35:1764-1772 \begin{tabular}{l|l|l} 
and Biochemistry & $\begin{array}{l}\text { DOI: 10.1159/000373988 } \\
\text { Published onlne: Mvarch 26, } 2015\end{array}$ & $\begin{array}{l}\text { O) 2015 S. Karger AG, Basel } \\
\text { www.karger.com/cpb }\end{array}$ \\
\cline { 2 - 3 }
\end{tabular}}

Wu et al.: FBXL5 Inhibits Snail1 in GC

\section{Disclosure Statement}

The authors have declared that no competing interests exist.

\section{References}

Zhao X, Li X, Yuan H: Micrornas in gastric cancer invasion and metastasis. Front Biosci 2013;18:803-810.

Liu G, Jiang C, Li D, Wang R, Wang W: Mirna-34a inhibits egfr-signaling-dependent mmp7 activation in gastric cancer. Tumour Biol 2014;35:9801-9806.

3 Mao D, Zhang Y, Lu H, Zhang H: Molecular basis underlying inhibition of metastasis of gastric cancer by anti-vegfa treatment. Tumour Biol 2014;35:8217-8223.

4 Ye Y, Zhou X, Li X, Tang Y, Sun Y, Fang J: Inhibition of epidermal growth factor receptor signaling prohibits metastasis of gastric cancer via downregulation of mmp7 and mmp13. Tumour Biol 2014;35:1089110896.

5 Shang Y, Cai X, Fan D: Roles of epithelial-mesenchymal transition in cancer drug resistance. Curr Cancer Drug Targets 2013;13:915-929.

6 Kiesslich T, Pichler M, Neureiter D: Epigenetic control of epithelial-mesenchymal-transition in human cancer. Mol Clin Oncol 2013;1:3-11.

7 Meng F, Wu G: The rejuvenated scenario of epithelial-mesenchymal transition (emt) and cancer metastasis. Cancer Metastasis Rev 2012;31:455-467.

8 Leroy P, Mostov KE: Slug is required for cell survival during partial epithelial-mesenchymal transition of hgf-induced tubulogenesis. Mol Biol Cell 2007;18:1943-1952.

-9 Lamorte L, Royal I, Naujokas M, Park M: Crk adapter proteins promote an epithelial-mesenchymallike transition and are required for hgf-mediated cell spreading and breakdown of epithelial adherens junctions. Mol Biol Cell 2002;13:1449-1461.

-10 Xiao X, Gaffar I, Guo P, Wiersch J, Fischbach S, Peirish L, Song Z, El-Gohary Y, Prasadan K, Shiota C, Gittes GK: M2 macrophages promote beta-cell proliferation by up-regulation of smad7. Proc Natl Acad Sci U S A 2014;111:E1211-1220.

11 Gregory PA, Bracken CP, Smith E, Bert AG, Wright JA, Roslan S, Morris M, Wyatt L, Farshid G, Lim YY, Lindeman GJ, Shannon MF, Drew PA, Khew-Goodall Y, Goodall GJ: An autocrine tgf-beta/zeb/mir-200 signaling network regulates establishment and maintenance of epithelial-mesenchymal transition. Mol Biol Cell 2011;22:1686-1698.

12 Valcourt U, Kowanetz M, Niimi H, Heldin CH, Moustakas A: Tgf-beta and the smad signaling pathway support transcriptomic reprogramming during epithelial-mesenchymal cell transition. Mol Biol Cell 2005;16:1987-2002.

13 Muthusami S, Prabakaran DS, Yu JR, Park WY: Egf-induced expression of fused toes homolog (fts) facilitates epithelial-mesenchymal transition and promotes cell migration in me180 cervical cancer cells. Cancer Lett 2014;351:252-259.

14 Sakuma K, Aoki M, Kannagi R: Transcription factors c-myc and cdx2 mediate e-selectin ligand expression in colon cancer cells undergoing egf/bfgf-induced epithelial-mesenchymal transition. Proc Natl Acad Sci U S A 2012;109:7776-7781.

15 Shirakihara T, Horiguchi K, Miyazawa K, Ehata S, Shibata T, Morita I, Miyazono K, Saitoh M: Tgf-beta regulates isoform switching of fgf receptors and epithelial-mesenchymal transition. EMBO J 2011;30:783795.

16 Ramos C, Becerril C, Montano M, Garcia-De-Alba C, Ramirez R, Checa M, Pardo A, Selman M: Fgf-1 reverts epithelial-mesenchymal transition induced by tgf-\{beta\}1 through mapk/erk kinase pathway. Am J Physiol Lung Cell Mol Physiol 2010;299:L222-231.

17 Billottet C, Tuefferd M, Gentien D, Rapinat A, Thiery JP, Broet P, Jouanneau J: Modulation of several waves of gene expression during fgf-1 induced epithelial-mesenchymal transition of carcinoma cells. J Cell Biochem 2008;104:826-839.

18 Yang N, Hui L, Wang Y, Yang H, Jiang X: Overexpression of sox2 promotes migration, invasion, and epithelialmesenchymal transition through the wnt/beta-catenin pathway in laryngeal cancer hep-2 cells. Tumour Biol 2014;35:7965-7973. 


\section{Cellular Physiology Cell Physiol Biochem 2015;35:1764-1772

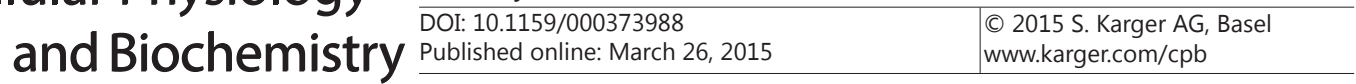 \\ Wu et al.: FBXL5 Inhibits Snail1 in GC}

19 Huang J, Xiao D, Li G, Ma J, Chen P, Yuan W, Hou F, Ge J, Zhong M, Tang Y, Xia X, Chen Z: Epha2 promotes epithelial-mesenchymal transition through the wnt/beta-catenin pathway in gastric cancer cells. Oncogene 2014;33:2737-2747.

20 Kaufhold S, Bonavida B: Central role of snail1 in the regulation of emt and resistance in cancer: A target for therapeutic intervention. J Exp Clin Cancer Res 2014;33:62.

-21 Franco DL, Mainez J, Vega S, Sancho P, Murillo MM, de Frutos CA, Del Castillo G, Lopez-Blau C, Fabregat I, Nieto MA: Snail1 suppresses tgf-beta-induced apoptosis and is sufficient to trigger emt in hepatocytes. J Cell Sci 2010;123:3467-3477.

-22 Zheng H, Shen M, Zha YL, Li W, Wei Y, Blanco MA, Ren G, Zhou T, Storz P, Wang HY, Kang Y: Pkd1 phosphorylation-dependent degradation of snail by scf-fbxo11 regulates epithelial-mesenchymal transition and metastasis. Cancer Cell 2014;26:358-373.

-23 Moroishi T, Yamauchi T, Nishiyama M, Nakayama KI: Herc2 targets the iron regulator fbxl5 for degradation and modulates iron metabolism. J Biol Chem 2014;289:16430-16441.

24 Ruiz JC, Bruick RK: F-box and leucine-rich repeat protein 5 (fbxl5): Sensing intracellular iron and oxygen. J Inorg Biochem 2014;133:73-77.

25 Ruiz JC, Walker SD, Anderson SA, Eisenstein RS, Bruick RK: F-box and leucine-rich repeat protein 5 (fbxl5) is required for maintenance of cellular and systemic iron homeostasis. J Biol Chem 2013;288:552-560.

26 Chollangi S, Thompson JW, Ruiz JC, Gardner KH, Bruick RK: Hemerythrin-like domain within f-box and leucine-rich repeat protein 5 (fbxl5) communicates cellular iron and oxygen availability by distinct mechanisms. J Biol Chem 2012;287:23710-23717.

27 Shu C, Sung MW, Stewart MD, Igumenova TI, Tan X, Li P: The structural basis of iron sensing by the human f-box protein fbxl5. Chembiochem 2012;13:788-791.

-28 Thompson JW, Salahudeen AA, Chollangi S, Ruiz JC, Brautigam CA, Makris TM, Lipscomb JD, Tomchick DR, Bruick RK: Structural and molecular characterization of iron-sensing hemerythrin-like domain within f-box and leucine-rich repeat protein 5 (fbxl5). J Biol Chem 2012;287:7357-7365.

29 Moroishi T, Nishiyama M, Takeda Y, Iwai K, Nakayama KI: The fbxl5-irp2 axis is integral to control of iron metabolism in vivo. Cell Metab 2011;14:339-351.

-30 Vinas-Castells R, Frias A, Robles-Lanuza E, Zhang K, Longmore GD, Garcia de Herreros A, Diaz VM: Nuclear ubiquitination by fbxl5 modulates snail1 DNA binding and stability. Nucleic Acids Res 2014;42:1079-1094.

31 Cen G, Ding HH, Liu B, Wu WD: Fbxl5 targets cortactin for ubiquitination-mediated destruction to regulate gastric cancer cell migration. Tumour Biol 2014;35:8633-8638.

-32 Liang CC, Park AY, Guan JL: In vitro scratch assay: A convenient and inexpensive method for analysis of cell migration in vitro. Nat Protoc 2007;2:329-333.

33 Lee MS, Kim S, Kim BG, Won C, Nam SH, Kang S, Kim HJ, Kang M, Ryu J, Song HE, Lee D, Ye SK, Jeon NL, Kim TY, Cho NH, Lee JW: Snail1 induced in breast cancer cells in 3d collagen i gel environment suppresses cortactin and impairs effective invadopodia formation. Biochim Biophys Acta 2014;1843:2037-2054. 